\title{
Teach the teacher
}

\section{Seminar zum Advanced Nursing Process - Rückblick und Ausblick}

Die Anwendung des neu etablierten und vertieften Pflegeprozesses inklusive Critical Thinking ist komplex, anspruchsvoll und muss trainiert werden. Acht Tage lang beschäftigten sich Pflegepersonen aus der Schweiz, Südtirol und Österreich im vergangenen Sommer in Salzburg mit der Entwicklung und Erweiterung ihrer diagnostische Kompetenz. Mit nachhaltigem Erfolg, wie die Evaluierung durch die Teilnehmer zum Ende des von Prof. Dr. Maria Müller-Staub und Claudia Leoni-Scheiber geleiteten Seminars ergab.

Die Fortschritte im Bereich der Informationstechnologien und pflegerischer Klassifikationssysteme sowie die Forderungen nach evidenzbasiertem Handeln haben zur Etablierung des Advanced Nursing Process (Ackley \& Ladwig, 2014) geführt.
„Ich erhielt viele Ideen für die Umsetzung. Ich habe mehr Sicherheit bekommen."

Dieser wurde wie folgt definiert: „Der vertiefte, fortgeschrittene Pflegeprozess besteht aus definierten, validierten Konzepten. Er umfasst Assessment, Pflegediagnosen, Pflegeinterventionen und Pflegeergebnisse und beruht aufwissenschaftlich basierten Pflegeklassifikationen" (MüllerStaub, Abt, Brenner, \& Hofer, 2014, S. 16). Die adäquate praktische Anwendung von Pflegeklassifikationen basiert auf klinischer Entscheidungsfindung, die Aspekte der diagnostischen Urteilsbildung und der therapeutischen wie ethischen Entscheidungsfindung beinhaltet (Gordon, 2008).

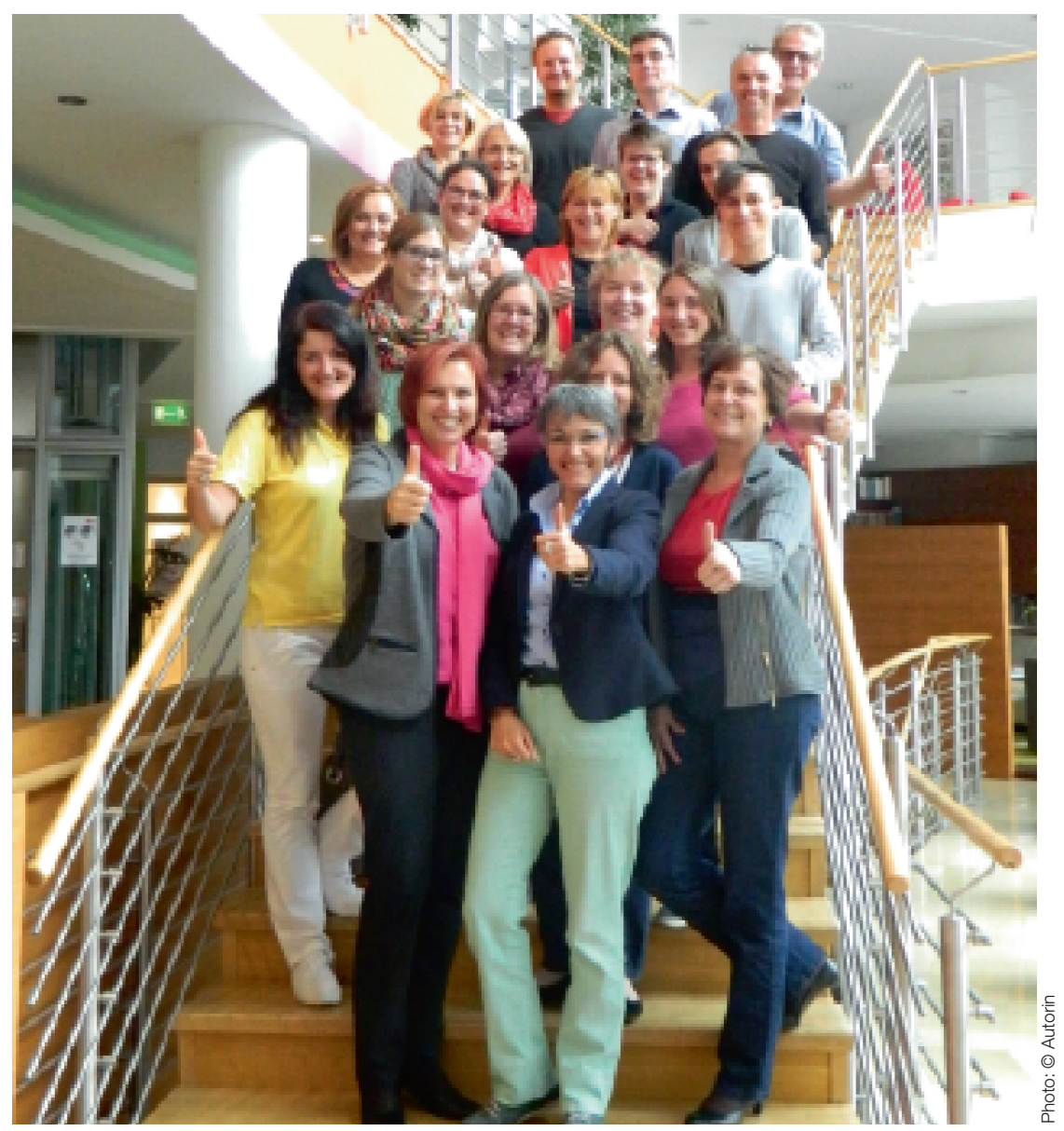

Die Seminarteilnehmer erhielten in acht Tagen ein breites Verständnis zu evidenzbasierten

Pflegeklassifikationen und deren Anwendung.
In Form von Vorträgen, Präsentationen, Fallbesprechungen, Selbststudienund Transferaufträgen erhielten die 21 Teilnehmer, sich zum Großteil aus Perso-

\section{„Das Seminar hat meine Sichtweise auf meinen Beruf und auf meine Arbeit geändert - Danke dafür!“}

nen mit Masterabschluss wie Pflegepädagogen, APNs sowie Pflegemanagern zusammensetzen, ein breites Verständnis zu evidenzbasierten Pflegeklassifikationen und deren Anwendung. Die Reflexion zum Seminarende zeigte äußerst zufrie-

„Caring trotz Wissenschaft: das hat mich sehr angesprochen. Die Professionalisierung stand auch immer im Vordergrund.“

dene Teilnehmende - zum Lernsetting gleichermaßen wie zu den Inhalten (www. pflege-pbs.ch/rueckmeldungen.html). Die Haltung zur Pflegediagnostik wie die Einstellung zum Beruf veränderten sich positiv. Ein solches Seminar und in dieser Intensität sei dringend nötig.

Im August 2017 findet das nächste achttägige Seminar in Salzburg statt.

Informationen: www.pflege-pbs.ch/weiterbildung. html.

Korrespondenz:

Claudia Leoni-Scheiber

E-Mail: c.leonischeiber@aon.at

Internet: www.pflege-pbs.ch

\section{LITERATUR}

Ackley, B. J., \& Ladwig, G. B. (2014). Nursing Diagnosis Handbook: An Evidence-based Guide to Planning Care (10th ed.). St. Louis: Mosby Elsevier.

Gordon, M. (2008). Assess Notes: Nursing assessment and diagnostic reasoning. Philadelphia: F.A. Davis.

Müller-Staub, M., Abt, J., Brenner, A., Hofer, B. (2014). Expertenbericht zum Verantwortungsbereich der Pflege. In: Schweizerischer Verein für Pflegewissenschaft (VFP) (Hrsg.). Bern: Schweizerischer Verein für Pflegewissenschaft (VFP). 